\title{
RÉSILIENCE DE L'AGGLOMÉRATION DE SFAX (TUNISIE MÉRIDIONALE) FACE AU CHANGEMENT CLIMATIQUE : ESSAI D'ÉVALUATION
}

\author{
Abdelkarim DAOUD ${ }^{1}$ et Salem DAHECH ${ }^{2}$ \\ ${ }^{1}$ Université de Sfax, Faculté des Lettres et Sciences Humaines - Laboratoire EEE \\ B.P. 553 - Sfax 3000 - Tunisie \\ daoudabdelkarim@yahoo.fr \\ ${ }^{2}$ Université de Sfax, Faculté des Lettres et Sciences Humaines - Laboratoire SYFACTE \\ Route de l'aéroport km 5, Sfax 3000 - Tunisie \\ salem.dahech@gmail.com
}

\begin{abstract}
Résumé
Dans l'agglomération de Sfax, située sur le littoral sud-oriental de la Tunisie, les températures minimales et maximales enregistrées entre 1950 et 2007 à la station située à l'aéroport indiquent une tendance significative au réchauffement. Cette période fut marquée aussi par l'occurrence des évènements pluviométriques extrêmes comme les inondations de 1969, 1982 et 2009. L'hypothèse principale de ce travail est que l'agglomération de Sfax présente une très faible résilience face aux effets du changement climatique. Pour l'étayer, les auteurs présentent dans une première partie, les manifestations du changement climatique, en s'appuyant sur les données fournies par l'Institut National de la Météorologie pour la période indiquée ci-dessus, insistant sur les facteurs favorisant ce réchauffement. Ils tentent ensuite d'établir la relation entre vulnérabilités et étalement de l'agglomération, particulièrement celles liées à l'îlot de chaleur urbain et à la pollution, au dysfonctionnement du système de transport et enfin, au risque d'inondation. Ces vulnérabilités sont d'autant plus aggravées que l'agglomération n'a établi aucune stratégie d'atténuation ou d'adaptation aux changements climatiques, ce qui diminue sa capacité de résilience. Les auteurs présentent ainsi dans une dernière partie, quelques pistes pour y remédier, et concluent sur la nécessité dans le futur, et avec l'avènement de la démocratie dans le pays, que les acteurs locaux, particulièrement les élus, et à travers une intercommunalité jusque là absente, tiennent compte du climat urbain et des changements climatiques dans la gestion de l'agglomération.
\end{abstract}

Mots-clés : Agglomération de Sfax, changements climatiques, aménagement, atténuation, adaptation, résilience.

\begin{abstract}
In the town of Sfax, located on the south-eastern coast of Tunisia, minimum and maximum temperatures recorded in the airport observatory between 1950 to 2007 indicate a significant warming trend. This period was also marked by the occurrence of extreme rainfall events such as floods of 1969, 1982 and 2009. The main working hypothesis in this paper is that the city of Sfax has very low resilience to climate change. At first, the authors present and explain the manifestations of climate change, based on data provided by National Institute of Meteorology for the period indicated above. At second, they try to establish the relationship between vulnerabilities and urban sprawl, particularly those linked to the urban heat island and pollution, related to dysfunction of the transport system and the flood risk. These vulnerabilities are compounded even more than the town has not established mitigation and adaptation strategy to climate change. Eventually, after the advent of democracy in the country, the authors propose, some ways to remedy the studied risks, and insist on taking into account the effect of climate change by policy makers in the future managements of the city.
\end{abstract}

The Sfax city climate change resilience (Southern Tunisia)

Keywords: Greater Sfax, climate change, planning, mitigation, adaptation, resilience.

\section{Introduction}

Le travail proposé prend comme cadre spatial d'étude l'agglomération de Sfax. Située sur le littoral Sud-Est de la Tunisie, cette agglomération compte aujourd'hui plus de 500000 habitants, sur une superficie urbaine d'environ 22000 ha. L'hypothèse principale de

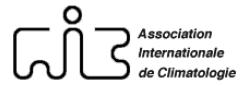


recherche est le constat que cette agglomération a enregistré au cours des cinq dernières décennies qui ont marqué sa forte évolution, une aggravation notable des vulnérabilités face aux changements climatiques et ses effets, et un affaiblissement de sa capacité de résilience. En effet, des études récentes montrent une tendance significative au réchauffement (évalué entre 1,5 et $2^{\circ} \mathrm{C}$ ) durant la période 1950-2007, avec une intensification et une extension de la saison chaude. De plus, l'occurrence des évènements thermiques exceptionnels a sensiblement augmenté (augmentation de 7 à $10 \mathrm{j} / \mathrm{an}$ du phénomène du sirocco), et même si on note une diminution du nombre de jours de pluie par an, les conséquences des évènements pluviométriques exceptionnels $(1969,1982,2009)$ prennent parfois des aspects catastrophiques.

Une rétrospective de la croissance urbaine de cette agglomération montre que l'étalement a commencé vers les années 1970, sacrifiant la proche banlieue de vergers-jardins typiques de la ville. L'étalement continue aujourd'hui dans le périurbain, créant une ceinture d'habitat souvent spontané, mal intégré dans la ville et parfois sur des sites à risques. Le grand projet de nouveau centre (Sfax-El-Jadida) sur les anciens cimetières jouxtant la médina, et la densification du bâti dans l'ancienne ville européenne, ont par ailleurs intensifié le phénomène de l'îlot de chaleur urbain (Dahech et al., 2011 ; Dahech et Beltrando, 2012). En outre, l'agglomération verra dans l'avenir la réalisation de deux méga projets urbains sur le littoral, le premier, en cours de réalisation, prévoit la dépollution du littoral Nord des anciennes usines de phosphate et du dépôt de phosphogypse et le remblaiement d'une partie des hauts fonds pour mettre en place une zone d'habitat et d'activités sur environ 400 ha. Le pendant de ce dernier, en cours d'étude, est prévu sur le littoral Sud de l'agglomération, aujourd'hui occupé principalement par une unité de transformation de phosphate, destinée à la délocalisation en 2011 suite à une décision prise en 2008, non suivie d'effet jusqu'à aujourd'hui. Il ne semble pas que ces projets aient prévu des mesures d'adaptation visant à améliorer la résilience de l'agglomération face aux effets des changements climatiques.

La prise en compte des changements climatiques et leurs effets est quasi absente dans les différents outils d'aménagement qui concernent l'agglomération. Ceci est un indicateur de la perception de ce phénomène par les acteurs de la ville, à différentes échelles. Toutefois, l'amélioration de la résilience de l'agglomération et de sa capacité d'adaptation passe par une meilleure connaissance scientifique des changements climatiques à des échelles fines. Leur prise en compte dans le Plan d'Aménagement Urbain actuellement en cours de révision pourrait prendre alors un aspect institutionnel à travers des stratégies d'atténuation ou d'adaptation, soit au niveau des choix d'aménagement ou des moyens de leur mise en œuvre.

Le travail proposé s'articule en trois parties. La première montre les manifestations du changement climatique dans l'agglomération de Sfax, la seconde tente de montrer les liens entre étapes et formes de la croissance urbaine et accroissement des vulnérabilités. Enfin, ce travail présente quelques éléments pour améliorer la résilience de l'agglomération face aux effets avérés ou attendus des changements climatiques.

\section{Méthodes et données}

Dans ce travail, nous traitons les températures minimales, maximales et moyennes journalières sous abri, ainsi que les moyennes décadaires, mensuelles et annuelles entre 1950 et 2007. Ces données sont utilisées pour étudier la variabilité interannuelle des températures et les tendances à différentes échelles temporelles afin de montrer l'extension et l'intensification de la saison chaude à la station de Sfax-El Maou, située dans l'aérodrome, à $6 \mathrm{~km}$ du centreville. Ensuite, nous utilisons le test d'homogénéité de Pettitt et de Lee, le test de détection de tendance de Man Kendall et la régression linéaire pour estimer la tendance. Puis, les données 
des précipitations quotidiennes sont traitées afin de montrer la variabilité interannuelle des totaux pluviométriques annuels et du nombre de jours de pluies. Les données météorologiques utilisées dans ce travail sont fournies par l'Institut National de la Météorologie de Tunisie. (INM).

La densification du tissu urbain ainsi que les activités humaines polluantes (trafic routier et pollution industrielle), ont contribué au réchauffement de la partie centrale de l'agglomération. De même, l'extension urbaine que celle-ci a connue durant les quatre dernières décennies a augmenté la vulnérabilité de la population face au risque d'inondation. Nous intégrons dans un Système d'Information Géographique, les surfaces bâties, déduites à partir d'une image satellitaire Ikonos (juin 2006), des photos aériennes de 1987 et de la carte topographique de 1955, les cours d'eau (oueds) et les aménagements de protection contre les inondations. Des analyses spatiales (croisement de couches et requêtes spatiales) permettent d'évaluer la vulnérabilité de l'agglomération face au risque d'inondation.

Nous nous basons sur un travail récent (Dahech, 2009) qui a montré à partir de mesures météorologiques (températures, humidité et vent), à l'échelle du quartier et de la rue, l'aggravation du réchauffement en milieu urbain à Sfax. Les résultats de ce travail sont étoffés par des observations de terrain réalisées en 2010 et 2011.

Nous partons de l'hypothèse que l'agglomération de Sfax présente aujourd'hui une faible résilience face aux effets des changements climatiques, du fait de l'absence d'une stratégie d'atténuation et d'adaptation. Nous observons les différents outils d'aménagement qui concernent l'agglomération (Schémas Directeurs d'Urbanisme et Plans d'aménagements) et nous verrons s'ils ont pris en compte les changements climatiques et leurs effets. Cela nous permettra d'évaluer la résilience de l'agglomération de Sfax face aux changements climatiques.

\section{Les manifestations du changement climatique dans l'agglomération de Sfax}

Des études récentes (Dahech, 2009 ; Daoud et Dahech, 2009 ; Dahech et Bouaziz, 2010) montrent une tendance significative au réchauffement durant la période 1950-2007, avec une intensification et une extension de la saison chaude. De plus, les évènements pluviométriques et thermiques exceptionnels qui se sont succédés durant les cinq dernières décennies semblent avoir des effets plus marqués, sur une ville devenue de plus en plus vulnérable.

\subsection{Une tendance significative au réchauffement}

Les températures minimales et maximales, enregistrées dans la station de Sfax-El Maou entre 1950 et 2007, ont connu une tendance significative à la hausse d'après le test de Man Kendall. Cette nette tendance significative à la hausse des températures se traduit par une très forte corrélation positive significative $(\mathrm{r}=0,75)$ entre les années et les températures moyennes. A Sfax, d'après l'équation de la régression linéaire, la température moyenne annuelle a augmenté de $0,033^{\circ} \mathrm{C} /$ an entre 1950 et 2007 . Cette hausse est plus importante au niveau des températures minimales soit $0,038^{\circ} \mathrm{C} /$ an contre $0,027^{\circ} \mathrm{C} /$ an pour les températures maximales (figure 1). Une rupture statistique est détectée au milieu des années 80 par les tests statistiques de pettitt et de Lee.

La hausse thermique observée depuis le début des années 1980 est conforme à celle détectée aux latitudes tempérées de l'hémisphère Nord, et particulièrement dans le bassin méditerranéen (Toreti et Desiato, 2008). A l'échelle régionale, la circulation générale justifie la hausse des températures : la fréquence du nombre annuel d'observations tri-horaires des

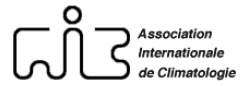


flux venant du sud est très corrélée avec les températures maximales $(r=0,7)$ pour la période 1950-2007. La même tendance est observée dans plusieurs stations tunisiennes comme à Tunis Carthage, Gabès et Gafsa (Hénia 2006 ; Dahech et Bouaziz, 2010).

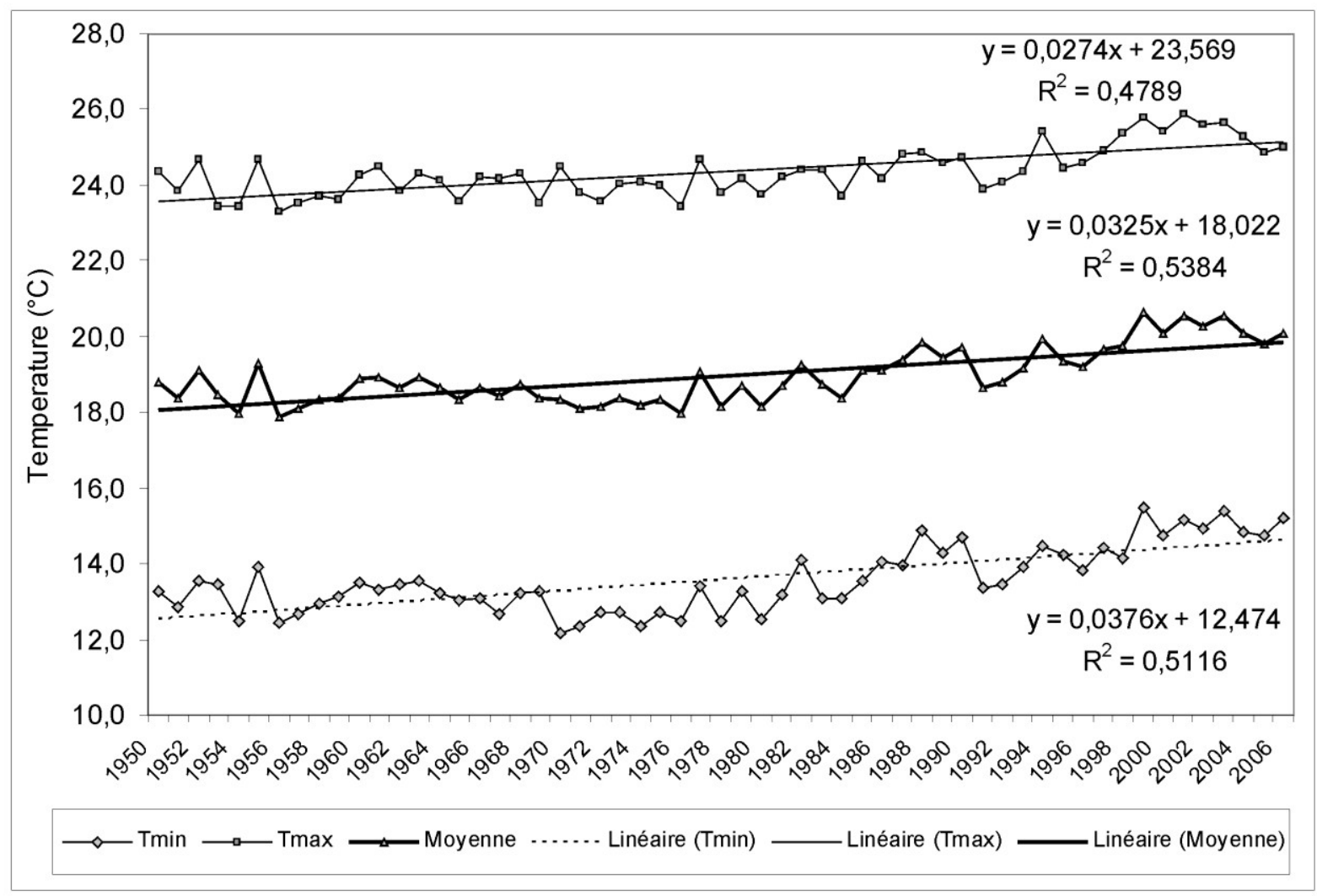

Figure 1 : Evolution des moyennes annuelles des températures minimales, maximales et moyennes entre 1950 et 2007 à Sfax (données : INM ; Dahech et Beltrando, 2012). Yearly variation of averaged, minimum and maximum temperature between 1950 to 2007 in Sfax (data from INM).

A l'échelle locale, l'urbanisation croissante que connaissent les agglomérations pourrait justifier une partie du réchauffement actuel (Mills, 2007). Les surfaces bâties modifient le bilan d'énergie prés du sol (Oke, 1987). Elles stockent plus de chaleur durant la journée (après artificialisation de la surface la quantité de chaleur sensible augmente au détriment de la chaleur latente) et la restituent la nuit sous forme de rayonnement tellurique. En effet, le rythme d'urbanisation dans l'agglomération de Sfax et en particulier autour de la station s'est accentué. Dans un rayon de $2 \mathrm{~km}$, la construction et l'extension des quartiers populaires denses 'El Bahri' au nord-est, 'El Khadra' à l'ouest et 'Erriadh' aux sud-est dès le début des années 1980 ont éventuellement amené cette hausse des températures (figure 2). Au début des années cinquante, le total des surfaces bâties situées dans un rayon de $2 \mathrm{~km}$ autour de la station météorologique n'excèdent pas un hectare. Ce chiffre est passé à environ 64 ha en 1987 et à 148 ha en 2006. Cette tendance a continué avec la construction d'un nouveau terminal dans l'aéroport de Sfax Thyna en 2008 et l'étalement urbain permanent que connaît l'agglomération. Parallèlement à cet étalement, dans les quartiers précités, le volume des bâtiments a augmenté. Selon nos observations, la plupart des habitations ont connu une extension verticale d'un à deux étages.

A l'échelle saisonnière, la hausse des températures est plus remarquable en été et au début de l'automne. Le temps estival se prolonge, désormais, jusqu'à la mi-octobre. En effet, les températures ont beaucoup augmenté durant les mois chauds (juin-septembre) par rapport aux mois relativement froids (décembre-février). 


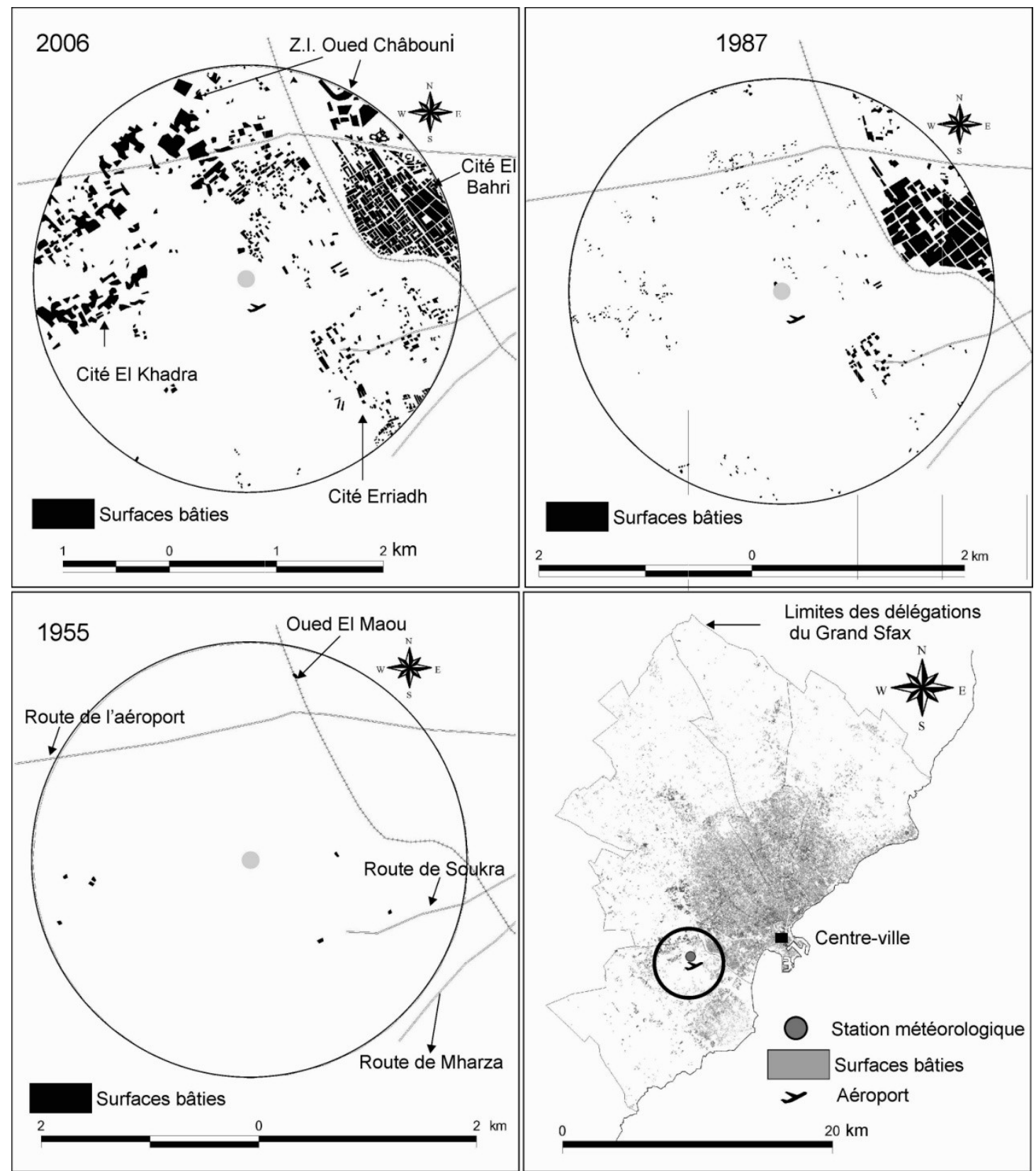

Figure 2 : Surfaces bâties situées dans un rayon de 2 kilomètres autour de la station météorologique Sfax El Maou en 1955, 1987 et 2006 (source : carte topographique de 1955, photographies aériennes de 1987 et image Ikonos datée de juin 2006). Built surfaces located at a radius of $2 \mathrm{~km}$ around the meteorological station "Sfax El Maou” in 1955, 1987 and 2006 (source: topographic map of 1955, aerial photographs of 1987 and Ikonos image recorded in June 2006).

Suivant la régression linéaire (figure 3), la hausse des températures minimales est significative aussi bien en hiver qu'en été. Elle est estimée à $0,44^{\circ} \mathrm{C} /$ décennie en été contre $0,27^{\circ} \mathrm{C} /$ décennie en hiver. La hausse des températures maximales est moins importante en été $\left(0,28^{\circ} \mathrm{C} /\right.$ décennie $)$ alors qu'elle n'est pas significative en hiver.

A l'échelle décadaire, les températures moyennes confirment cette intensification de la saison chaude depuis le début des années 1980. Des décades avec des températures moyennes dépassant $30^{\circ} \mathrm{C}$ sont devenues plus fréquentes, particulièrement au mois d'août à Sfax (figure 4). Parallèlement, les températures au début de l'automne passent de $24^{\circ} \mathrm{C}$, durant les deux premières décades du mois de septembre entre 1950 et 1980 , à plus de $26^{\circ} \mathrm{C}$ durant les deux dernières décennies (figure 4). 

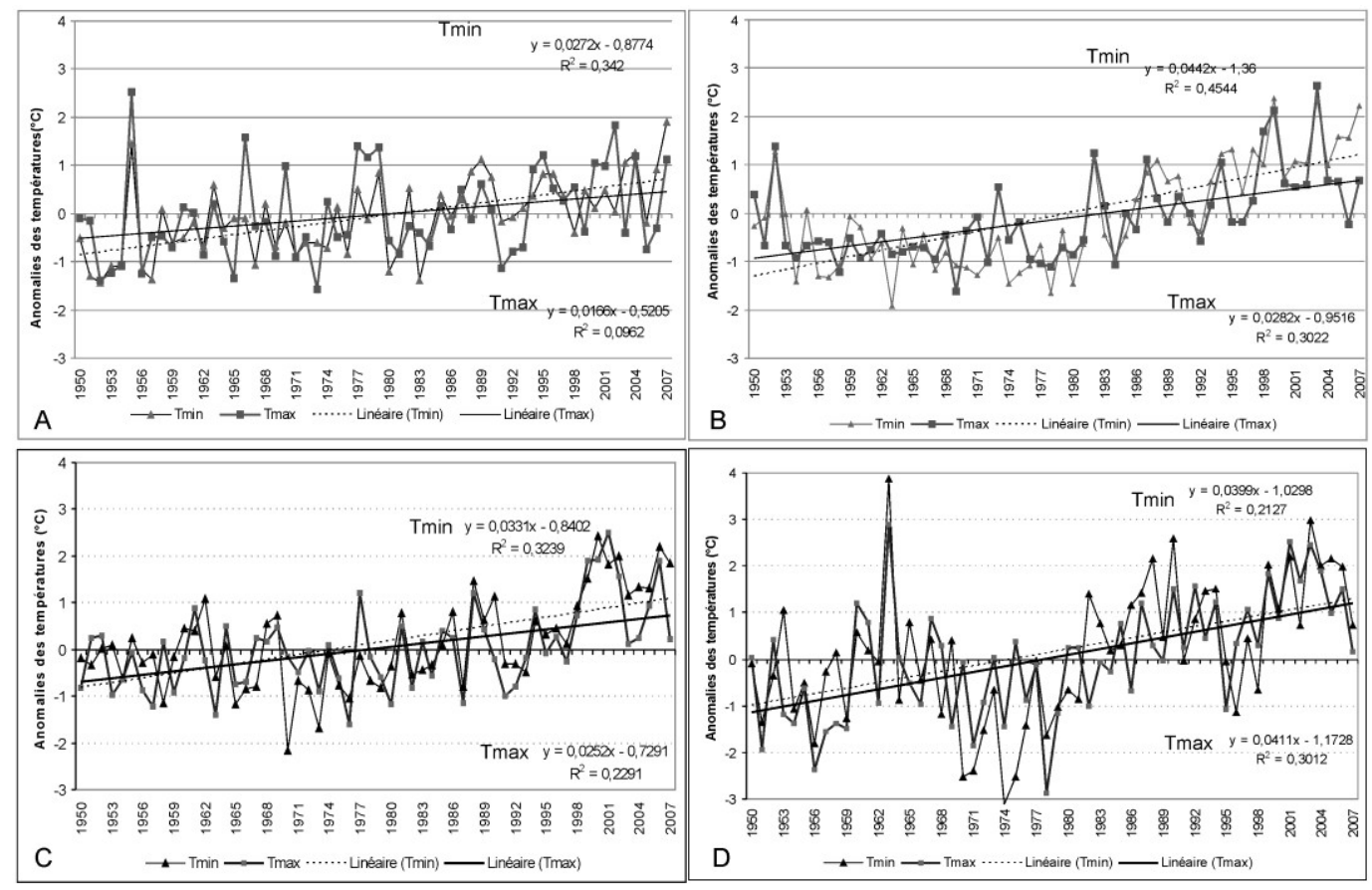

Figure 3 : Variabilité interannuelle des anomalies des températures minimales et maximales durant A) l'hiver (moyenne de décembre, janvier et février), B) l'été (juin, juillet, août et septembre), C) du printemps (mars, avril et mai) et de D) l'automne (octobre et novembre) ; d'après des données de l'INM. Seasonal variability of minimum and maximum temperatures: A) during winter (average of December, January and February), B) summer (average June, July, August and September), C) spring (March, April and May) and D) autumn (October and November); data from INM.

Figure 4 : Variabilité interannuelle des températures moyennes décadaires à Sfax entre 1950 et 2007 (source : Données INM ; Dahech, 2009). Interannual variability of decadal mean temperatures in Sfax. Source: NMI, 1950-2007 (Dahech, 2009).

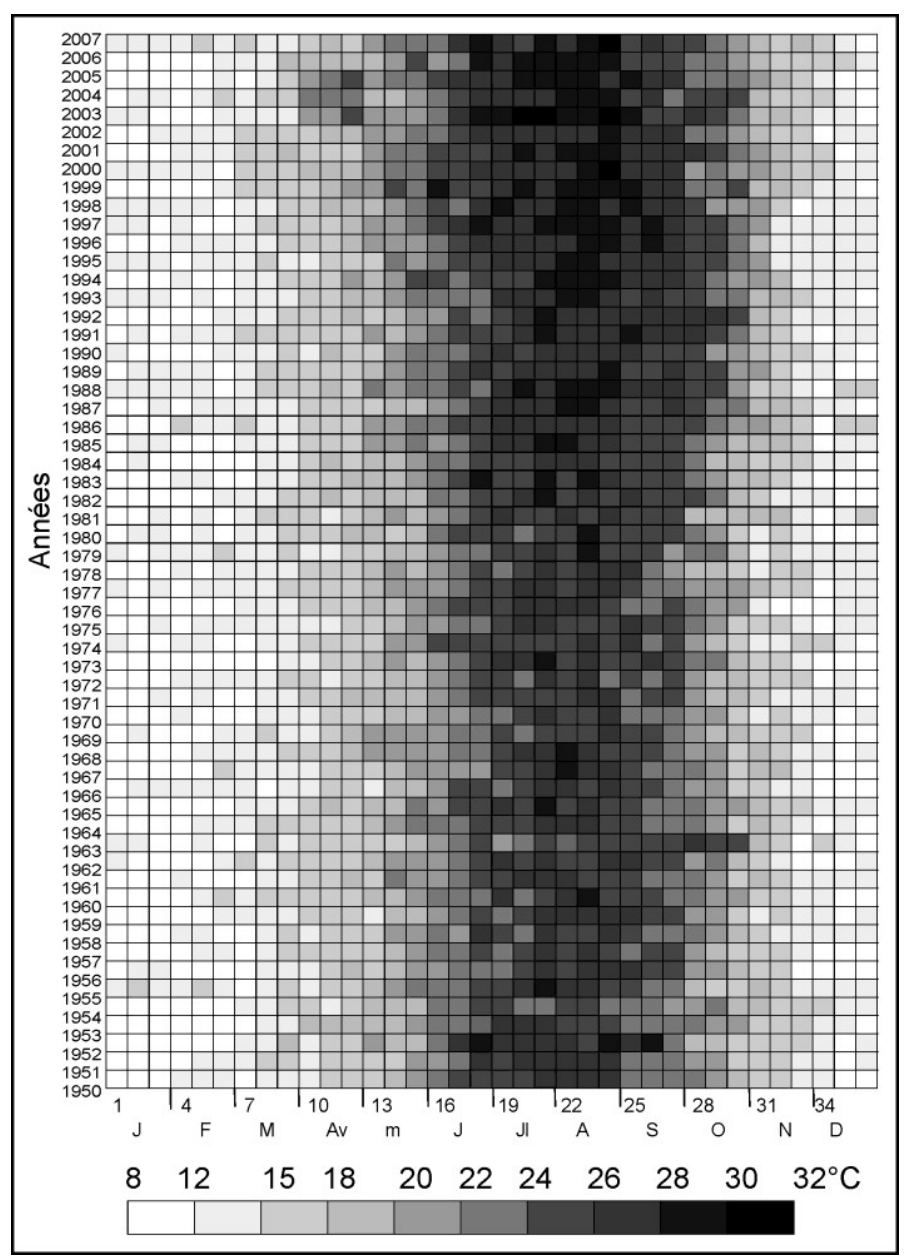


L'extension et l'intensification de la saison chaude s'expliquent, en partie, par une augmentation de l'occurrence des situations extrêmes. En effet, durant les mois de juin, juillet, août et septembre, entre 1950 et 2007 , le nombre de jours durant lesquels les températures minimales et maximales dépassaient le seuil du centile 95 enregistre une tendance à la hausse. Les températures maximales, enregistrées durant les mois de juin, juillet, août et septembre et dépassant $36^{\circ} \mathrm{C}$, soit 5 à $7^{\circ} \mathrm{C}$ supérieurs à la moyenne mensuelle sur la période (1950-2007), connaissent une tendance positive significative. En effet, durant la période 1950-1980, le mercure n'avait dépassé $36^{\circ} \mathrm{C}$ que durant environ 5 jours/an contre environ 15 jours/an entre 1980 et 2007 (figure 5). En dehors des moyennes, nous notons certaines situations extrêmes ; à titre d'exemple, en 2003 , le mercure a dépassé $36^{\circ} \mathrm{C}$ durant 28 jours. En outre, en fin d'été et au début de l'automne, la chaleur excessive peut s'accompagner d'un taux d'humidité élevé, d'où une amplification de l'inconfort thermique (Dahech et Bouaziz, 2010).

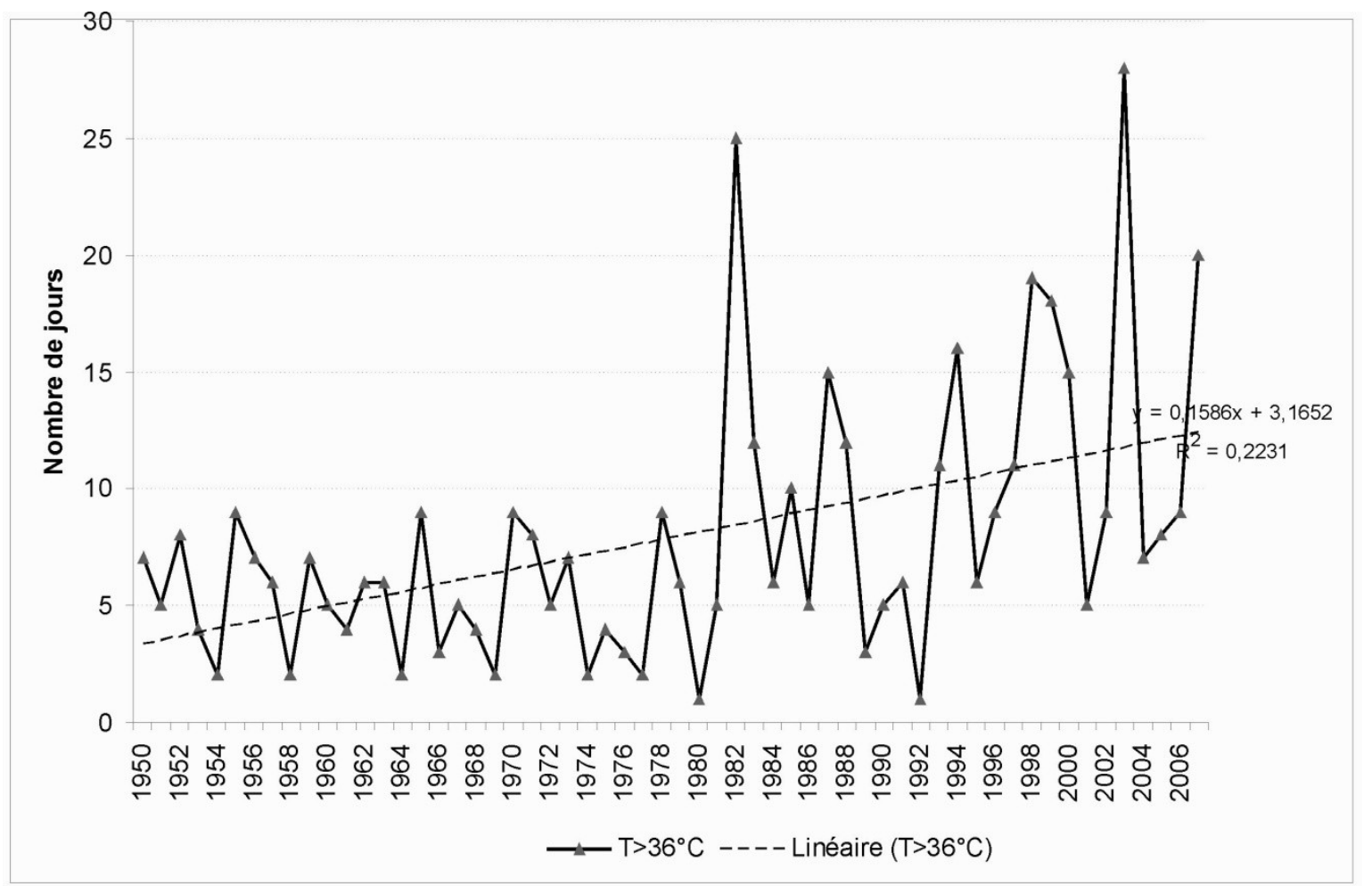

Figure 5 : Variabilité annuelle du nombre de jours durant lesquels la température maximale a dépassé $36^{\circ} \mathrm{C}$ entre 1950 et 2007 (données de l'INM). Annual variability in the number of days when the maximum temperature exceeded $36^{\circ} \mathrm{C}$ between 1950 to 2007 (data from INM).

\subsection{Pas de tendance des précipitations mais des inondations graves probables}

Le total pluviométrique annuel (figure 6A) et le nombre annuel de jours de pluies (figure 6B) enregistrés dans l'aérodrome de Sfax entre 1950 et 2007 ne montrent pas de tendances significatives. Cependant, l'agglomération de Sfax peut être menacée par des inondations catastrophiques. Ce fut le cas le 7 octobre 1969 (156 mm), le 27 octobre 1969 (127 mm), le 30 octobre $1983(141 \mathrm{~mm})$ et le 23 septembre 2009 (103 $\mathrm{mm}$ en $35 \mathrm{~min}$ au centre-ville). Survenant souvent en automne suite à une cyclogenèse sur le golfe de Gabès, les effets de ces averses torrentielles sont d'autant plus destructeurs que la vulnérabilité de l'agglomération est de plus en plus aggravée. A titre indicatif, les inondations de 1969 (qui intéressèrent également la Tunisie centrale) causèrent des pertes humaines et des dégâts matériels considérables ; celles de 1982, bien que plus localisées, emportèrent un millier d'habitations et une centaine de personnes, et celles de 2009 paralysèrent la zone centrale de la ville de Sfax pendant plusieurs heures. 
Figure 6 : Variabilité interannuelle du total pluviométrique (A) et du nombre de jours de pluies (B) enregistrés dans l'aérodrome de Sfax entre 1950 et 2007 (données de l'INM). Variability of total annual rainfall (A) and number of rainy days $(B)$ recorded in the airport of Sfax between 1950 to 2007 (Data from INM).

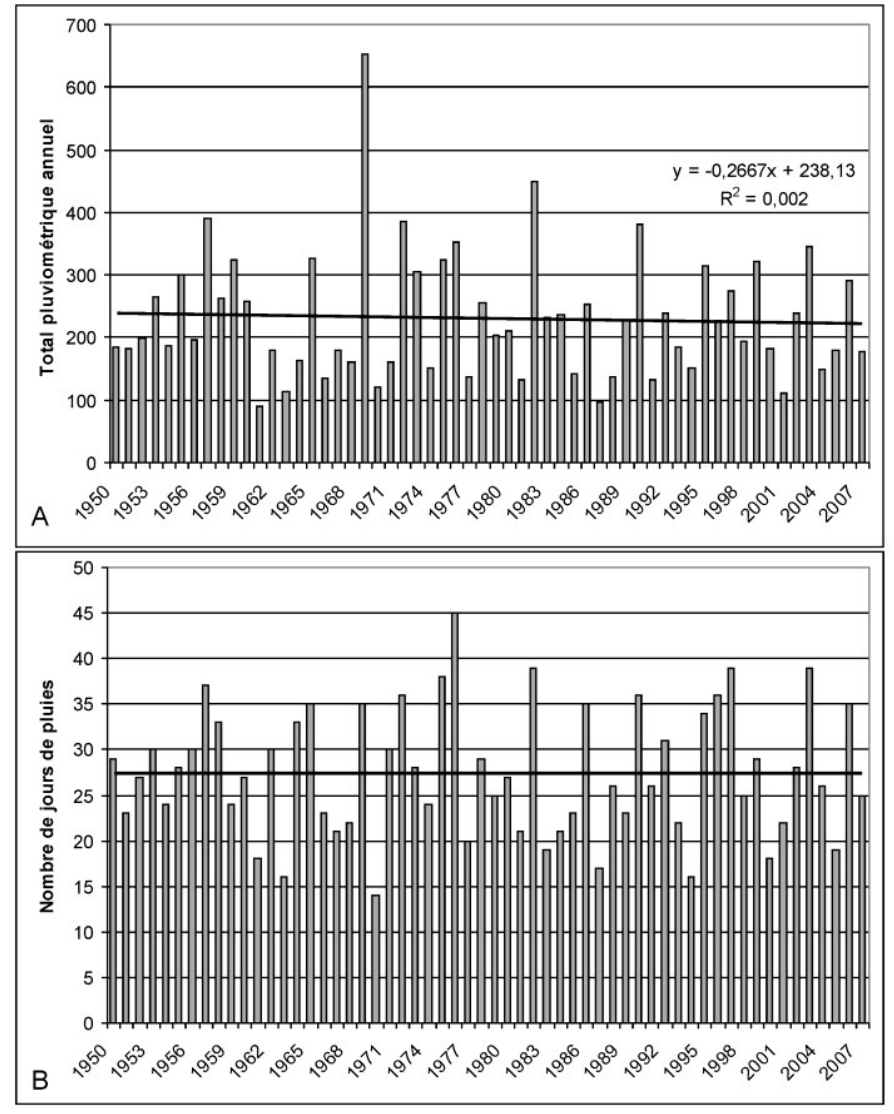

\section{Une agglomération de plus en plus étalée et vulnérable}

\subsection{Etalement urbain et dysfonctionnements de la ville}

Historiquement, Sfax fut à l'origine une médina arabo-musulmane typique, construite au IX $^{\text {ème }}$ siècle et entourée de remparts, qui se dressent jusqu'à aujourd'hui, couvrant une superficie de 24 hectares. Sans se hasarder à faire une rétrospective historique détaillée de l'étalement de la ville sur son arrière-pays, il nous semble néanmoins important de noter que les phases les plus significatives de cet étalement eurent lieu au $\mathrm{XX}^{\text {ème }}$ siècle. Ainsi, avec la colonisation, une ville européenne allait naître dès le début du siècle, et s'étaler progressivement à l'Est de la médina, sur des terrains gagnés sur les hauts-fonds. Des éléments structurants allaient apparaître à la même période, comme le port (le quai des phosphates fut inauguré en 1907), la voie ferrée qui le relie au bassin phosphatier de Gafsa et la route qui relie Sfax à Tunis. Les années 1930 virent l'apparition des premiers faubourgs (appelés Rbats), accueillant les flux de migrants, accentués avec la crise économique de cette période. Outre ses fonctions économiques, la médina assurait une fonction résidentielle pour la population sfaxienne. L'arrière-pays, constitué à l'époque de vergers-jardins (appelés jneins), dans lesquels étaient disséminées des habitations typiques (appelées borjs) servait aussi de lieu de résidence estivale pour une partie de la population. Le milieu du XX ${ }^{\text {eme }}$ siècle vit d'importantes opérations de transformation du littoral (Megdiche, 2010), avec en particulier l'installation d'industries très polluantes, comme celles des unités de transformation des phosphates, comme la SIAPE en 1952 et la NPK dès les premières années de l'indépendance. Une véritable rupture spatiale va s'opérer à partir des années 1970 lorsque l'étalement urbain touche de façon systématique la ceinture des vergers, qui subit des morcellements continus, entrainant une densification du bâti et de zone de résidence saisonnière, devenant une zone d'habitat permanent, d'aspect pavillonnaire (Karray, 1982 ; Megdiche, 1985 ; figure 7). 


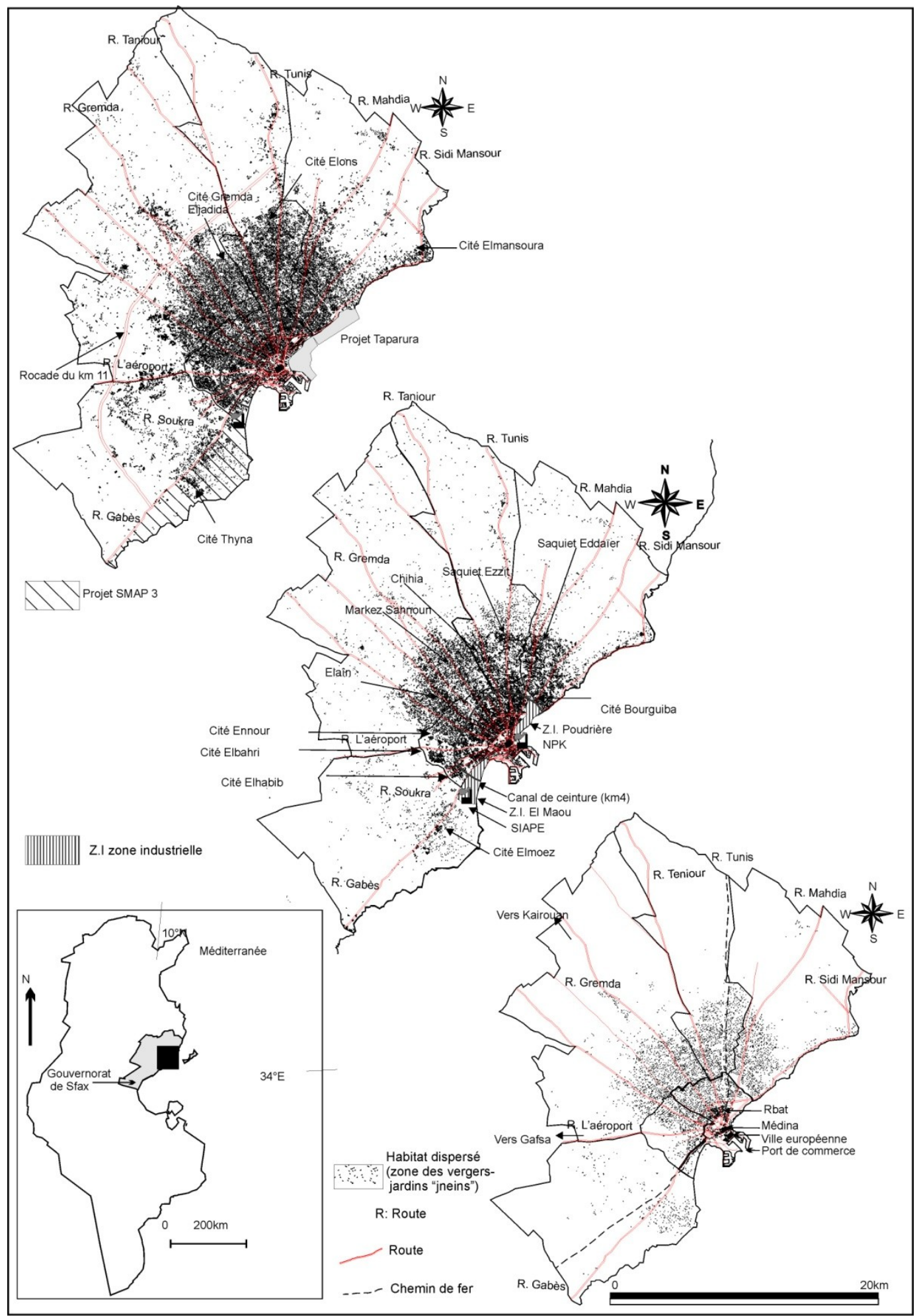

Figure 7 : Les grandes étapes de l'étalement urbain de l'agglomération de Sfax. De haut en bas : 2006, 1987 et 1955. The main stages of the urban sprawl of the city of Sfax (from top to bottom: 2006, 1987 and 1955).

Dans la conjoncture d'ouverture et de libéralisme économique qui avait prévalu dès le milieu des années 1970, le littoral de l'agglomération allait abriter l'essentiel des zones industrielles de la ville, en conformité avec les orientations du Plan Directeur d'Urbanisme de 1977. Les deux dernières décennies virent le renforcement de cette tendance à l'étalement de la ville (Bennasr, 2003), sans commune mesure avec le poids démographique de l'agglomération, ainsi que la densification de la zone centrale et péricentrale par affinage 
urbain, et la prolifération de l'habitat anarchique dans les secteurs périurbains (Baklouti, 2004, 2010).

L'étalement urbain est à l'origine de plusieurs dysfonctionnements et problèmes environnementaux dans l'agglomération de Sfax. En effet, la concentration des activités tertiaires dans les zones centrales et péricentrales, ou industrielles sur le littoral, ont généré des mouvements pendulaires de la population résidant dans la zone d'habitat pavillonnaire et dans la périphérie. En l'absence de modes de transports publics efficients, ces mouvements s'effectuent par des moyens privés, principalement la voiture et les deux roues motorisées. Outre les pertes de temps et le gaspillage d'énergie qu'ils génèrent, ces déplacements quotidiens sont à l'origine de pollutions diverses, responsables de l'aggravation de l'îlot de chaleur urbain. De plus, l'industrialisation littorale a provoqué des dégradations parfois irréversibles des eaux marines et continentales. L'urbanisation anarchique dans les secteurs périurbains a entrainé l'aggravation des risques d'inondation de quartiers entiers situés en bordures d'oueds ou en zones d'expansion des crues.

\subsection{Des vulnérabilités multiples et de plus en plus aggravées : le cas de l'îlot de chaleur urbain}

Le dernier rapport du GIEC précise que d'ici la fin du XXI ${ }^{\text {ème }}$ siècle, les épisodes climatiques extrêmes deviendront plus fréquents : vagues de chaleur, inondations catastrophiques (GIEC, 2007). Pour l'agglomération de Sfax, l'impact des modes d'urbanisation sur le climat urbain a été démontré à travers les températures plus élevées, enregistrées dans les zones centrales denses, par rapport au reste de l'agglomération (Dahech, 2009). La densification du bâti dans le centre (qui comprend la médina, la ville européenne et Sfax-El-jadida) et la rareté des espaces verts (MEAT, 1998) ont aggravé l'îlot de chaleur urbain. D'après l'image Ikonos prise le 7 juin 2006 (résolution métrique) et les travaux de terrain réalisés en mars 2011, les espaces verts ne représentent que $1 \%$ de la superficie totale du centre-ville, soit environ 26 ha (figure 8).
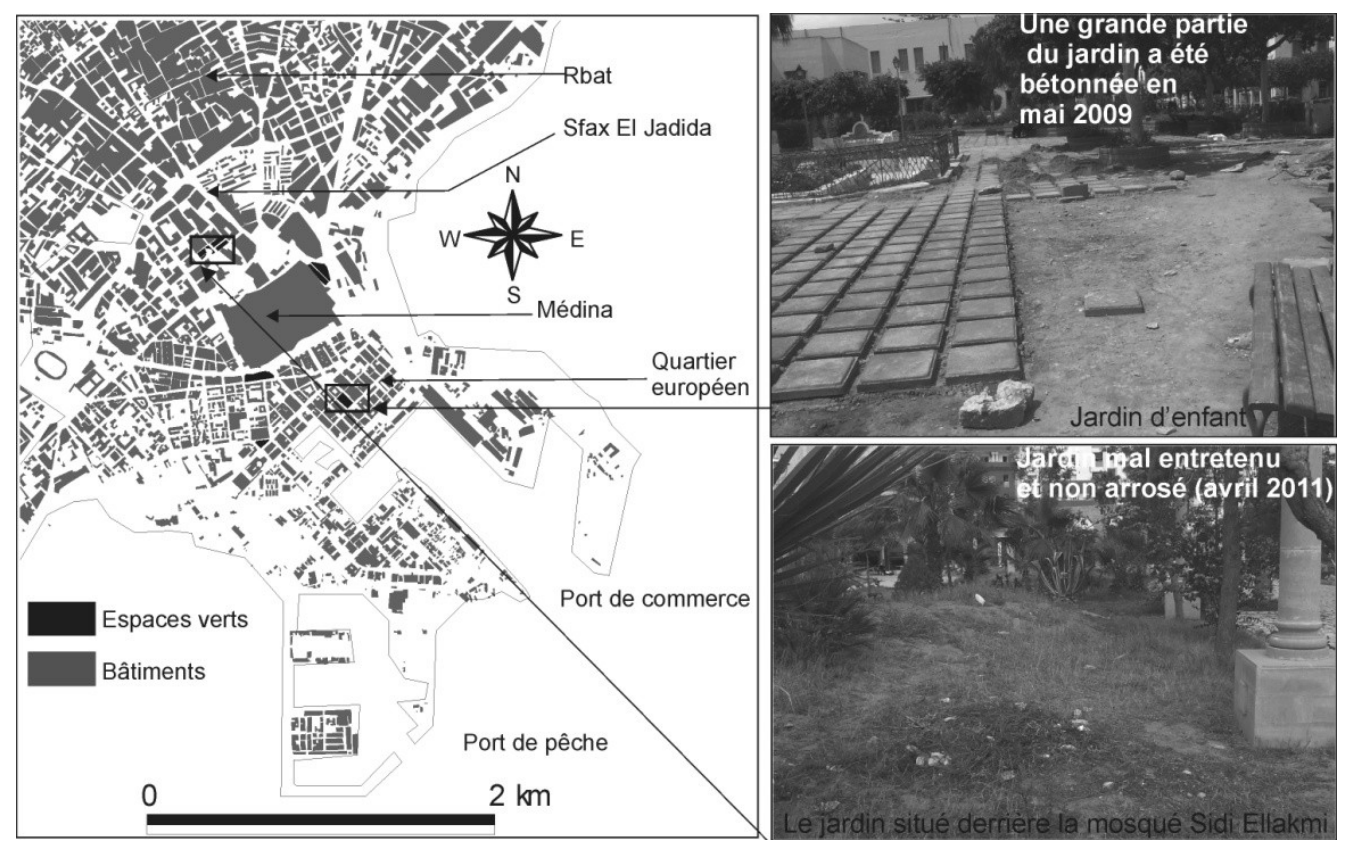

Figure 8 : Répartition des espaces verts dans le centre-ville de Sfax (fond : image Ikonos de 2006) et exemple du jardin d'enfant (en haut à droite) et du jardin de la mosquée Ellakhmi (en bas à droite, clichés : S. Dahech). Distribution of green space in downtown area of Sfax (bottom, Ikonos image 2006) and example of kindergarten (top right) and the garden of the mosque Ellakhmi (in bottom right, snapshots S. Dahech). 
De plus, certaines caractéristiques de l'urbanisme moderne, appliquées aux nouvelles constructions d'immeubles dans le centre, ainsi que les matériaux choisis, favorisent la surchauffe des bâtiments. Les bâtiments avec des parois vitrées sont difficiles à rafraîchir en été, d'où une consommation d'énergie relativement élevée.

Une étude menée par Dahech (2009) a mis en exergue l'impact de ces bâtiments sur la température. Cette étude fut basée sur des mesures des températures intérieures par six capteurs de types "tinytalk" enregistrant en continu la température de l'air avec un pas de temps de 30 minutes. Ces capteurs furent installés dans des pièces de bureaux d'une superficie comparable $\left(10 \mathrm{~m}^{2}\right)$ au centre-ville (figure 9) durant le mois de juillet 2008. Les sites de mesures furent sélectionnés suivant le type de parois et l'exposition au soleil. Durant les jours non ouvrables (pas de climatisation), un écart d'environ $5^{\circ} \mathrm{C}$ fut enregistré en moyenne entre des bureaux orientés vers la même direction, les premiers avec des façades vitrées et les seconds avec des façades opaques "classiques" (figure 9). L'écart était plus creusé au niveau des températures maximales, puisqu'il atteignit $15^{\circ} \mathrm{C}$ (Dahech, 2009). Dans les pièces à façade vitrée, l'amplitude thermique diurne était relativement élevée et les pics thermiques variaient en fonction de l'exposition au soleil et de l'angle d'incidence alors que les températures étaient quasiment constantes dans les bâtiments à façades opaques (points 5 et $6)$.
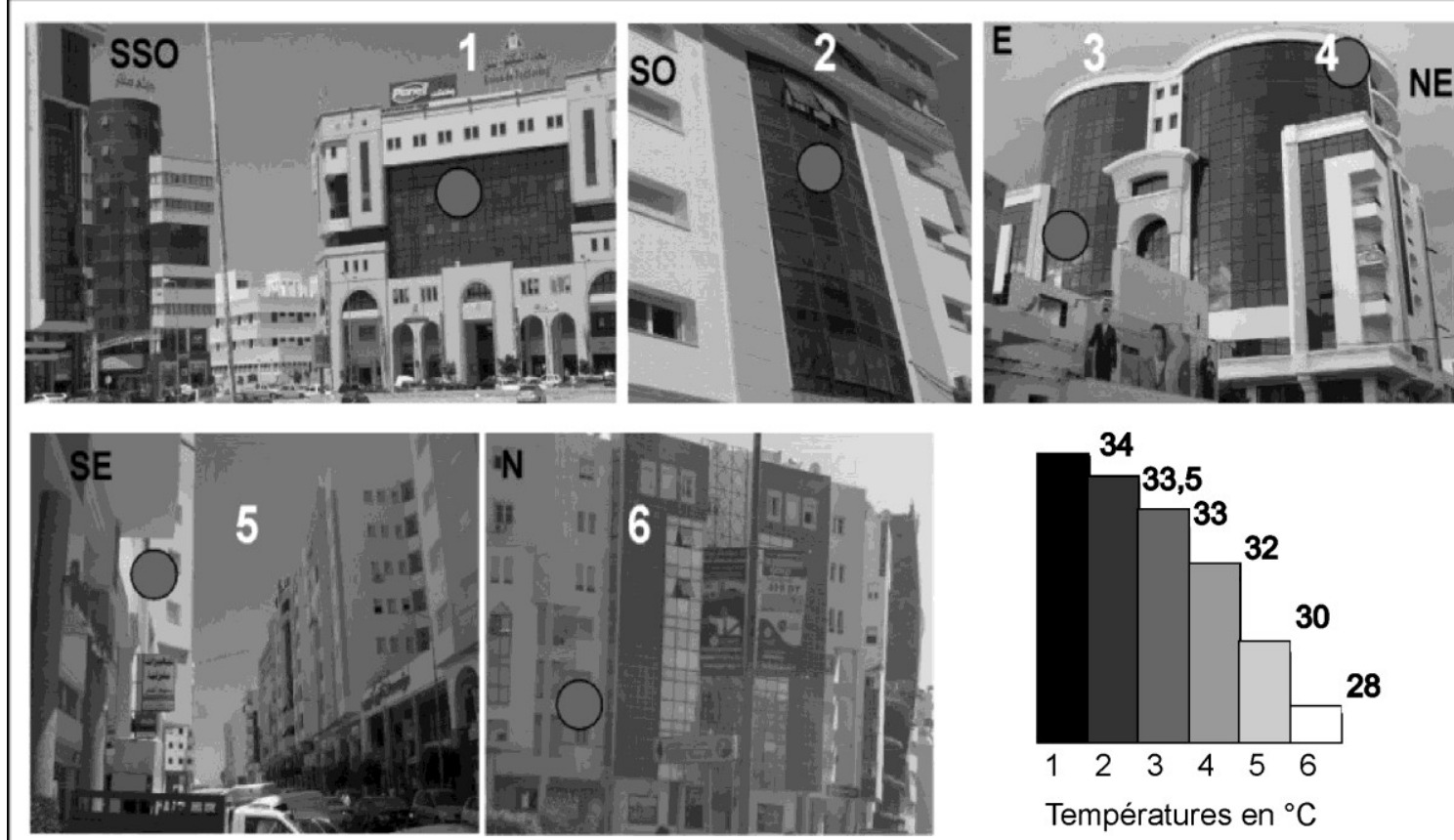

Températures en ${ }^{\circ} \mathrm{C}$

Figure 9 : Températures quotidiennes moyennes enregistrées à l'intérieure de 6 bureaux au centre-ville de Sfax (capteurs tinytalk, moyennes des 4 dimanches du mois de juillet, pas de temps $30 \mathrm{~min}$ ); source : Dahech, 2009. Daily average of inside temperatures recorded in 6 rooms in Sfax downtown (tinytalk sensors, averages of four Sundays of July; source: Dahech, 2009).

Les bâtiments avec des façades vitrées, considérés comme signe de modernité et de haut standing, fleurissent partout à Sfax. Nous estimons que les $2 / 3$ des bâtiments de Sfax El Jadida possèdent des façades vitrées, à l'instar du complexe immobilier Elaïn Centre, exposé Sud (figure 10). De surcroît, certains bâtiments officiels présentent aussi le même aspect, à l'instar de la Caisse Nationale de l'Assurance Maladie (CNAM) à Sfax El Jadida. La fièvre des parois vitrées a touché aussi les anciens bâtiments qui ont procédé à des travaux de rénovation de leurs façades en posant les vitres sur les murs. C'est le cas de l'hôtel Donia ou de la Clinique Essalama (figure 10). 


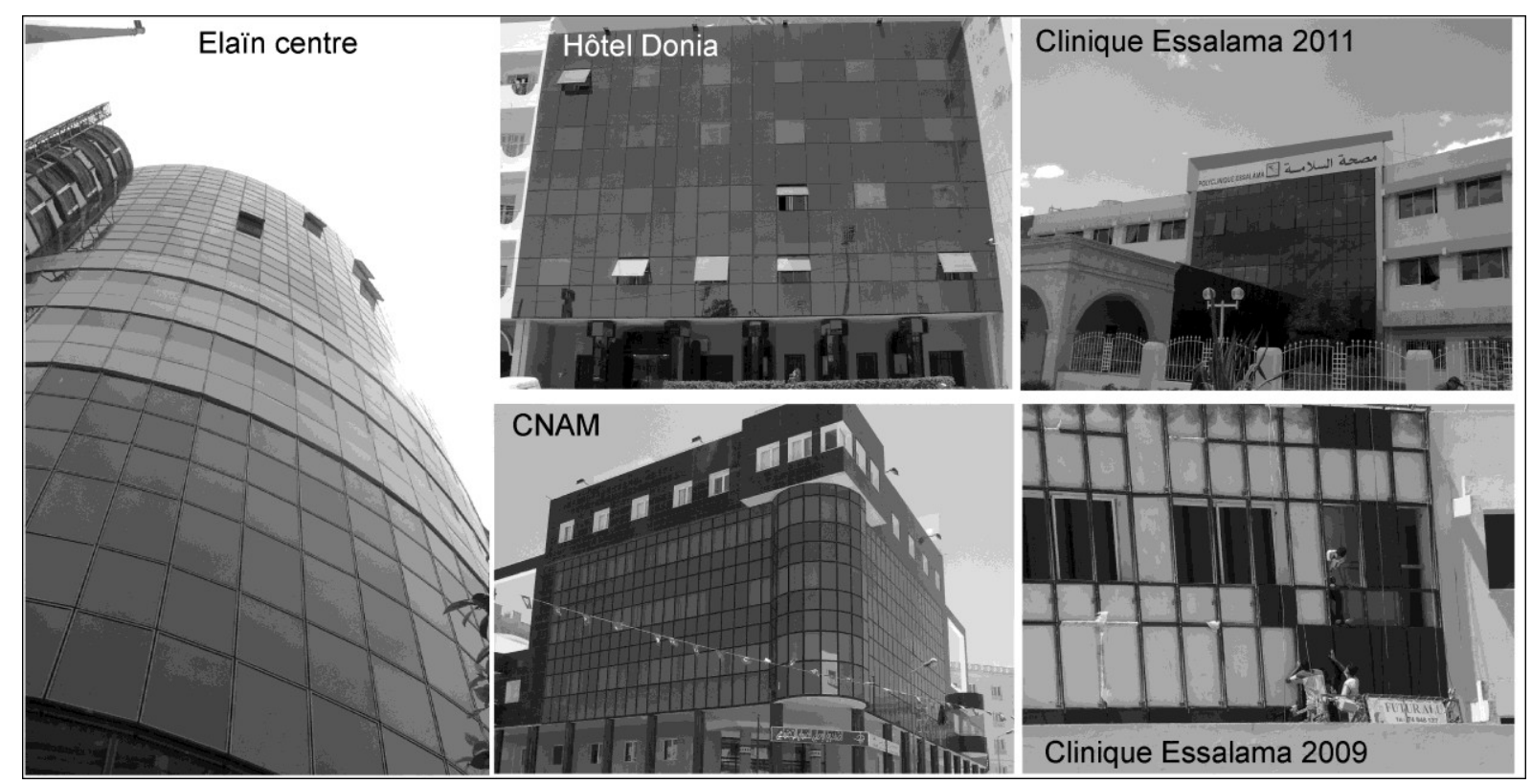

Figure 10 : Exemple de bâtiments vitrés à Sfax (Clichés : S. Dahech, 2009 et 2011). Example of glazed buildings in Sfax (Dahech pictures recorded in 2009 and 2011).

\subsection{Une agglomération de plus en plus vulnérable aux risques d'inondation}

Malgré l'imposante infrastructure de protection contre les inondations mise en place depuis 1984, à la suite de la catastrophe causée par les inondations d'octobre 1982, l'agglomération de Sfax est encore vulnérable face aux inondations, comme l'a montré l'évènement de septembre 2009. Cette vulnérabilité a évolué dans le temps et dans l'espace, en fonction de l'étalement de l'agglomération et de son fonctionnement. En effet, si les infrastructures mises en place après 1982 (principalement le canal de ceinture) furent destinées à assurer la protection de la partie centrale et ses environs jusqu'à un rayon de 4 kilomètres environ, les nouvelles zones urbanisées dans la périphérie de l'agglomération, dans des secteurs à risque, ne bénéficient souvent d'aucune protection. Cette urbanisation a entraîné l'imperméabilisation de superficies de plus en plus importantes et l'augmentation des coefficients de ruissellement lors des évènements pluvieux. En effet, la superficie du bâti en amont du canal de ceinture (appelé ceinture Bourguiba) est passée de 2350 ha en 1987 à 3900 ha en 2006. De plus, plusieurs habitations ont occupé les lits des oueds, puisque la superficie du bâti situé à moins de $20 \mathrm{~m}$ des lits des cours d'eau non endigués a doublé en 2006 par rapport à 1987, passant de 180 à 355 ha, particulièrement à proximité des oueds Chaâbouni, Ezzit et Sidi Salah, à environ $13 \mathrm{~km}$ du centre-ville (figure 11).

A leur vulnérabilité physique, s'ajoute une vulnérabilité sociale et économique, puisque ces zones sont souvent occupées par des catégories sociales à revenus fort modestes. Toutefois, les inondations de septembre 2009, qui ont vu un orage localisé déverser sur le centre-ville une quantité de $103 \mathrm{~mm}$ en 35 minutes (entre 12 h et 12 h 35 environ), ont révélé de nouvelles vulnérabilités. En effet, cet orage est survenu à une heure de pointe pendant laquelle la circulation automobile dans le centre et aux grands carrefours connait une forte congestion, en rapport avec les flux considérables de voitures quittant le centre vers la périphérie. Des bouchons interminables se sont formés au moment de l'orage, bloquant les automobilistes dans leurs véhicules, qu'ils étaient obligés de quitter une fois submergés par plus de un mètre d'eau. Il est clair que les dysfonctionnements du système de transport urbain, et particulièrement l'absence de transports publics efficients (Municip-Sfax, 2004), sont à l'origine de cette nouvelle vulnérabilité du centre-ville. 


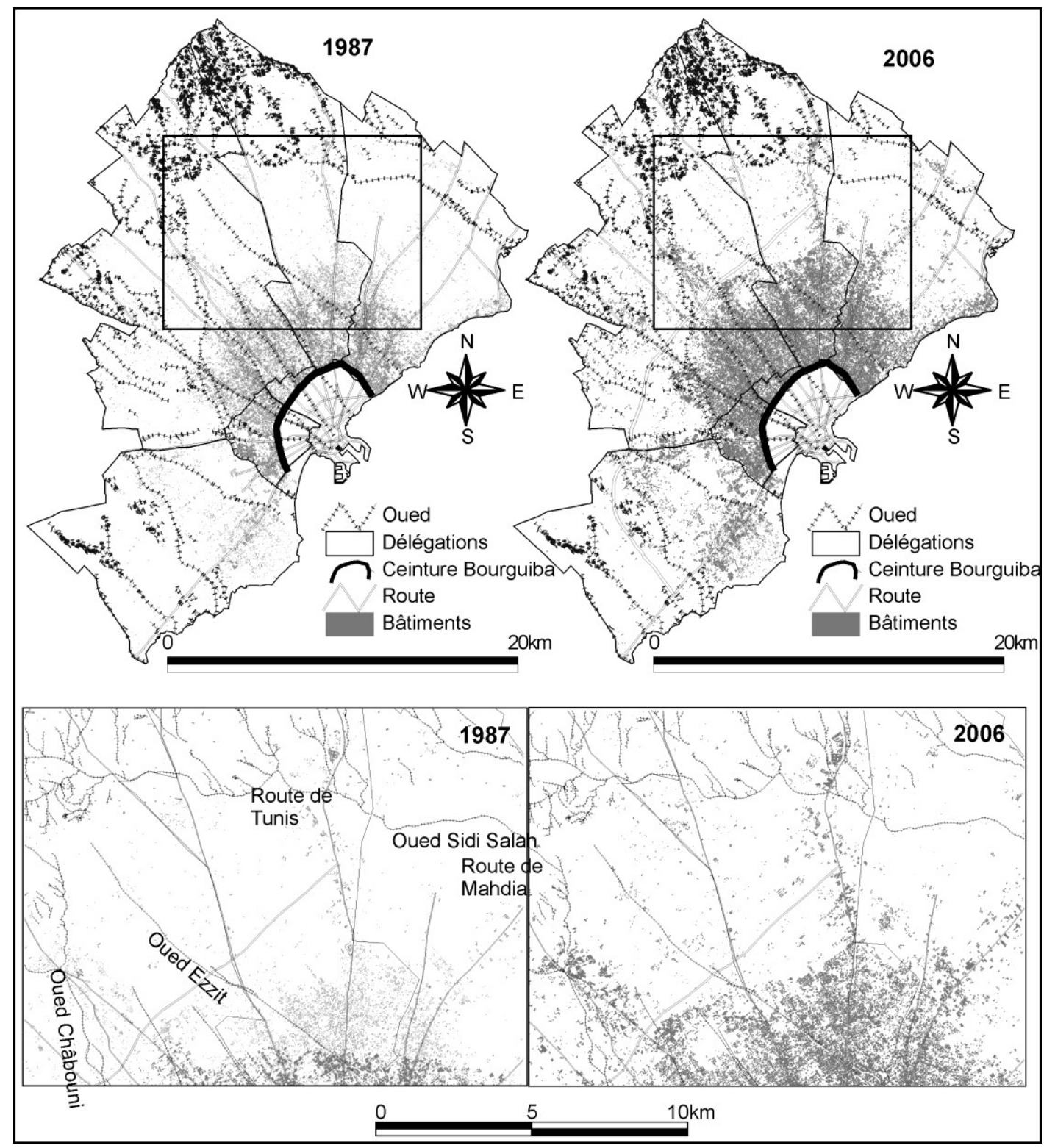

Figure 11 : Bâti et réseau hydrographique dans la partie non protégée par le canal de ceinture (canal du km 4$) \mathrm{du}$ Grand Sfax en 1987 et 2006 (bâti déterminé à partir des photographies aériennes de 1987 et d'une couverture Ikonos datée de 2006 ; cours d'eau tracés à partir des cartes topographiques de 1992 au 1/25000). Built area and river system in the unprotected part of Grand Sfax in 1987 and 2006 (built areas determined from the aerial photos of 1987 and Ikonos image of 2006. Wadi are plotted from topographic maps of 1992 with 1/25000 scale).

\section{Quelle résilience face aux changements climatiques?}

\subsection{Place des changements climatiques dans la politique environnementale en Tunisie}

Bien que les villes du Sud soient globalement plus vulnérables au changement climatique que celles du Nord, les stratégies d'adaptation et d'atténuation y sont quasi-absentes faute de moyens humains, techniques et matériels. De plus, le débat sur l'amélioration de la résilience des villes et les stratégies de sa mise en œuvre dans les pays du Nord (à l'instar des Plans Climat Energie Territoriaux en France) n'ont aucune commune mesure avec la situation dans les pays du Sud (ADEME, 2009). Toutefois, la Tunisie publie, depuis une quinzaine d'années environ, un rapport annuel intitulé Etat de l'environnement, comportant des rubriques fixes comme la gestion des ressources naturelles, la lutte contre la pollution et l'amélioration du cadre de vie ou l'activité des différents secteurs économiques. Ce n'est qu'en 2009 qu'un 
chapitre d'une vingtaine de page sur les changements climatiques fut inséré dans la rubrique activités économiques et développement durable. Ce chapitre (Medd, 2009a) note qu'en 2009, l'étude d'une stratégie nationale d'adaptation au changement climatique fut démarrée, incluant les composantes thématiques, la mise en œuvre ainsi que le cadre institutionnel, le suivi et l'évaluation. Cette stratégie concerne la gestion des ressources en eau, la lutte contre la désertification et l'érosion marine, la gestion des déchets, la protection contre les inondations, la mise en place d'un système de veille climatique et d'alerte précoce, etc.

A l'échelle locale, dans les documents d'aménagement (comme les Schémas Directeurs d'Aménagement ou les Plans d'Aménagement Urbains), les changements climatiques ne sont pas perçus comme facteurs déterminants devant infléchir de façon profonde et significative la conception des choix d'aménagement et les prescriptions que ces documents, vers de nouvelles stratégies territoriales, incluant atténuation et adaptation. Toutefois, il faut dire que le Schéma Directeur d'Aménagement du Grand Sfax (1998) avait préconisé une grande opération de dépollution de l'agglomération, et parmi les solutions retenues, figuraient l'aménagement du littoral nord (projet Taparura) et la mise en place d'un Parc National Protégé sur le littoral sud, dans la zone humide de Thyna. Notons aussi qu'en 2002, la commune de Sfax avait initié une méga-étude sur les prospectives de développement pour 1'horizon 2016. Toutefois, cette étude, achevée en 2009, ne contient aucune mention explicite relative à l'adaptation au changement climatique ou à l'atténuation de ses effet (Daoud et Dahech, 2009), bien que parmi les objectifs du projet figurent ceux de faire de Sfax "une métropole méditerranéenne compétitive, de haute technologie, durable, solidaire, attractive et réconciliée avec son littoral. » (Megdiche 2010).

\subsection{Quelles pistes pour l'atténuation et l'adaptation à Sfax ?}

A l'échelle locale, il n'y a pas véritablement une conscience chez les acteurs de la ville de la nécessité d'adopter une stratégie d'adaptation aux effets du changement climatique. D'ailleurs, sur le plan national, il n'y a pas une stratégie nationale, un cadre d'action permettant d'orienter et de cadrer les actions locales à l'échelle des communes. Les études et rapports publiés par le Ministère de l'Environnement (Medd, 1999a et b) présentent des mesures ou des études visant à améliorer la résilience des écosystèmes, ou des activités économiques comme l'agriculture ou le tourisme, mais pas encore la ville en tant que système.

L'adaptation et l'atténuation passent par la réduction des vulnérabilités, mais pour le cas de Sfax comme pour les autres grandes villes tunisiennes, il n'y a pas de programmes spécifiques pour évaluer les vulnérabilités urbaines face au changement climatique, car l'application de toute mesure, même ponctuelle, devrait être précédée par un diagnostic et une évaluation scientifique des résultats préconisés. Concernant l'îlot de chaleur urbain, il est dommage de constater qu'aucune mesure n'est prise, aussi bien en ce qui concerne l'urbanisme ou les matériaux utilisés, au moment même où la densification des zones centrales et péricentrales continue, avec des façades non adaptées, et où l'étalement progresse de façon démesurée à la périphérie de l'agglomération. Dans ce volet de limitation des effets de l'îlot de chaleur urbain, la stratégie reste à inventer et à mettre en exécution, mais devrait tenir compte de quelques principes fondamentaux : limiter au maximum l'utilisation des façades en verre, en particulier pour les bâtiments administratifs, profiter des effets des brises dans le choix des orientations, privilégier de manière absolue les transports en commun dans l'accès au centreville et multiplier les rues piétonnes et les espaces végétalisés. La mise en œuvre de ces principes à l'échelle de l'agglomération nécessite le développement de l'intercommunalité, entre la commune de Sfax et les sept communes périphériques, et dont l'ensemble forme le Grand-Sfax. Dans le cadre de la décentralisation attendue au lendemain de la révolution en 
Tunisie, l'intercommunalité pourrait constituer un garant supplémentaire de bonne gouvernance et d'amélioration de la capacité d'adaptation, à travers l'amélioration des compétences humaines et techniques et des conditions d'accès au marché des capitaux et des technologies. La mise en œuvre de ces principes pourrait nécessiter une priorisation selon des critères établis. Concernant la réduction de la vulnérabilité face aux risques d'inondation, il est important de faire de la carte des risques et vulnérabilités un outil d'aménagement, ce qui revient à lui conditionner la décision d'urbaniser. Pour le centre-ville, la vulnérabilité peut se trouver considérablement atténuée suite à la mise en place de plans de gestion de situation de crise, incluant la limitation du trafic automobile et sa déviation en cas de besoin (cas de Nice par exemple).

\subsection{Repenser les grands projets d'aménagement en cours, pour une meilleure résilience}

D'après ce qui a précédé, nous utilisons le concept de résilience dans le sens de l'adaptation de la ville, par son organisation, son aménagement, son urbanisme, ses activités de production ou de loisirs et les modes de vie et de consommation de ses habitants, au changement climatique et aux enjeux environnementaux qui lui sont liés. L'amélioration de la résilience peut s'inscrire dans le moyen et le long terme. Ceci confirme l'idée que les villes se situent au cœur du changement climatique (Damon, 2011). A ce titre, les grands projets d'aménagement en cours dans l'agglomération de Sfax pourraient constituer une assise exceptionnelle à l'adaptation. En effet, le projet d'aménagement du littoral nord de Sfax, connu sous 1'appellation de projet Taparura, pourrait être repensé dans certaines de ses composantes, pour intégrer les principes d'atténuation et d'adaptation face au changement climatique. Globalement, ce projet vise à "réconcilier Sfax avec la mer » à travers la dépollution du littoral (interdit à la baignade depuis 1977) par le confinement du dépôt de phosphogypse laissé par l'usine de traitement des phosphates NPK, le remblaiement d'une bande de quelques centaines de mètres de largeur sur un linéaire de quatre kilomètres environ, permettant de gagner environ 420 hectares de terrains constructibles. Ce projet gagnerait à appliquer un urbanisme adapté aux fortes chaleurs, bannissant les façades vitrées et profitant de l'orientation à même de favoriser la circulation de la brise marine et la ventilation naturelle, d'autant plus que le projet est en début de réalisation (phase dépollution et remblai). Il gagnerait aussi à privilégier le transport en commun dans ses liaisons avec le centre actuel ainsi que le transport en site propre (Municip-Sfax, 2004).

Le pendant sud du projet Taparura est le projet appelé SMAP 3, visant la dépollution du littoral sud et la réaffectation de la friche industrielle que laisserait l'usine de transformation des phosphates (SIAPE) après sa délocalisation. L'aménagement durable de ce littoral pourrait adopter les principes d'adaptation au changement climatique retenus sur le site du projet Taparura. Dans les deux cas, les décideurs locaux à l'échelle des collectivités pourraient s'inspirer des quartiers durables ou des écoquartiers, construits sur les friches urbaines des pays industrialisés comme il en existe en Allemagne, aux Pays-Bas ou dans les pays scandinaves, en les adaptant aux conditions climatiques locales dans leur architecture et les matériaux utilisés. Ces quartiers privilégient le cadre de vie à la rentabilité, préconisent l'économie d'espace, d'énergie ainsi qu'en transport individuel en vue de la réduction de "l'impact écologique global». Ces quartiers prônent des modes de vie écologiques surtout concernant l'habitat et la mobilité. En effet, les écoquartiers peuvent être un moyen parmi d'autres pour construire une ville durable, car ils peuvent constituer une nouvelle politique urbaine de la ville, visant une meilleure maîtrise de l'énergie et une limitation de l'utilisation de la voiture en milieu urbain. Il est vrai que certaines infrastructures urbaines, comme les transports publics en site propre, l'utilisation de certains matériaux de construction, économes en énergie, peuvent présenter des coûts élevés au moment de leur mise en place, mais sur le long terme, ils permettront des économies. Mais sans un engagement de la totalité des

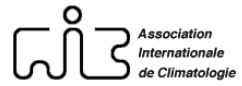


habitants pour des comportements pro-environnementaux au quotidien, les écoquartiers ne seraient que des îlots isolés. L'action des élus locaux peut s'inspirer aussi de ce qui est appelé en France le Plan Climat-Energie-Territorial (ADEME, 2009). Ce plan pourrait s'établir, d'après l'ADEME selon les étapes suivantes : la préfiguration (organisation, prédiagnostic...), le diagnostic et mobilisation (diagnostic climatique, concertation), la construction $d u$ plan (actions immédiates, scénarios prospectifs) et la mise en ouvre. L'évaluation s'effectuant à tous les stades.

\section{Conclusion}

La tendance vers le réchauffement ne laisse plus de doute aujourd'hui dans l'agglomération de Sfax. A terme, cela conduira à accroitre les vulnérabilités urbaines, face auxquelles Sfax présente aujourd'hui une faible résilience. Pour être efficaces, les mesures d'atténuation et d'adaptation devraient présenter une cohérence dans les niveaux décisionnels, de l'Etat aux collectivités locales. Néanmoins, l'échelle locale peut s'avérer très efficace dans les prises de décision en vue de l'atténuation ou de l'adaptation, pour réduire la vulnérabilité des populations face au changement climatique. L'opportunité s'offre aujourd'hui pour l'agglomération de Sfax de mettre en œuvre des mesures efficaces d'adaptation et d'atténuation dans les grandes opérations d'aménagement du littoral. Si au stade actuel, le projet Taparura d'aménagement du littoral Nord de Sfax a réussi à dépolluer la côte et gagner 420 hectares de terrains urbanisables, l'enjeu est désormais d'y mettre les jalons d'une ville durable, économe en espace et en énergie, adaptée par son urbanisme et son mode de vie au quotidien au changement climatique. Dans ce cas, le projet en cours d'étude, d'aménagement du littoral sud, devrait constituer le vrai pendant du littoral nord. Ainsi, l'agglomération de Sfax, qui a longtemps tourné le dos à la mer en la sacrifiant pour une industrialisation et un mode d'urbanisation peu soucieux d'environnement, verrait son littoral la conduire dans l'avenir vers la durabilité. Mais au préalable, décideurs locaux et population doivent faire montre d'un degré de sensibilisation au changement climatique, à même de les conduire à tenir compte du climat urbain dans l'élaboration concertée des documents d'aménagement. La révision en cours du Schéma Directeur d'Aménagement du Grand-Sfax pourrait en donner l'exemple pour l'ensemble de l'agglomération.

\section{Bibliographie}

AGENCE DE L'ENVIRONNEMENT ET DE LA MAITRISE DE L'ENERGIE (ADEME), 2009 : Construire et mettre en cuvre un plan-climat territorial. Guide méthodologique. 228 pages.

BAKLOUTI A., 2004 : Les quartiers périurbains du Grand-Sfax, formation, fonctionnement et aménagement. Thèse de Doctorat de géographie, Université de Tunis I, Faculté des Sciences Humaines et Sociales.

BAKLOUTI A., 2010 : Dynamique résidentielle dans les espaces ruraux périurbains des villes tunisiennes : cas du Grand Sfax. Climats, Société et dynamique des paysages ruraux en Tunisie. Coord. Cherif A. et Hénia L., Université de Tunis, Faculté des Sciences Humaines et Sociales, 292-318.

BENNASR A., 2003 : L'étalement urbain de Sfax. Revue Tunisienne de Géographie, 35, 4988.

CANTAT O., 2004 : L'îlot de chaleur urbain parisien selon les types de temps. Norois, 191 (2), 75-105. 
CARREGA P., 1994 : Topoclimatologie et habitat. Revue d'Analyse Spatiale Quantitative et Appliquée, 35 et 36, 408 pages.

DAHECH S. et BELTRANDO G., 2005: Etude multiscalaire de la répartition spatiotemporelle du monoxyde de carbone à Sfax: impact de la circulation automobile et de l'industrie. Journées interdisciplinaires de la qualité de l'air $-3^{\text {ème }}$ édition, 30 juin- ${ }^{\text {er }}$ juillet 2005, 18 pages.

DAHECH S., BELTRANDO G., BIGOT S., 2005 : Utilisation des données NOAA-AVHRR dans l'étude de la brise thermique et de l'Ilot de chaleur à Sfax (sud-est tunisien). Cybergéo, 317,19 pages.

DAHECH S., 2009 : Aggravation du réchauffement en milieu urbain à Sfax (Tunisie). Fifth Urban Research Symposium 2009, Marseille, 27 juin-1 ${ }^{\text {er }}$ juillet, www.urs2009.net/docs/papers/Dahech.pdf.

DAHECH S. et BOUAZIZ R., 2010 : Extension de la saison chaude et situations d'inconfort thermique extrêmes en fin de l'été et au début de l'automne en Tunisie. XXIII ${ }^{\text {ème }}$ Colloque de l'Association Internationale de Climatologie, Rennes, 4-9 septembre 2010, 149-154.

DAHECH S., 2011 : Evolution de la répartition spatiale des températures de l'air et de surface dans l'agglomération de Sfax (1987-2010) et impact sur la consommation d'énergie durant la saison chaude. Colloque 'Renforcer la résilience au changement climatique des villes : du diagnostic spatialisé aux mesures d'adaptation', Metz, 7-8 juillet 2011.

DAHECH S., DAOUD A. et BELTRANDO G., 2011 : Les inégalités spatiales de la qualité de l'air dans l'agglomération de Sfax et ses environs. Le cas des températures, de la brise, du $\mathrm{CO}$ et du $\mathrm{SO}_{2}$. Cybergéo.revue.org/24701.

DAHECH S. et BELTRANDO G., 2012 : Observed temperature evolution in the city of Sfax (Middle Eastern Tunisia) for the period (1950-2007). Climatic Change, DOI 10.1007/s10584012-042-x.

DAMON J., 2011 : Villes à vivre. Modes de vie urbains et défis environnementaux. Odile Jacob. 279 pages.

DAOUD A. et DAHECH S., 2009 : Changements climatiques et gouvernance urbaine : cas de l'agglomération de Sfax (Tunisie méridionale). Fifth Urban Research Symposium 2009, Marseille 27 juin- $1^{\text {er }}$ juillet, 12 pages, www.urs2009.net/docs/papers/Daoud.pdf.

GROUPE D'EXPERTS INTERGOUVERNEMENTAL SUR L'EVOLUTION DU CLIMAT (GIEC), 2007 : Rapport de synthèse sur les changements climatiques. Genève, OMM, 114 pages.

KARRAY N., 1982 : Le Grand-Sfax : Evolution récente, développement futur. Thèse d'Etat, Université Paris I, 2 volumes, 277 et 228 pages + annexes.

MEGDICHE T., 1985 : Les processus de la croissance urbaine dans la périphérie de Sfax. Thèse de troisième cycle en Géographie et Aménagement, Université de Toulouse Le Mirail, 272 pages.

MEGDICHE T., 2010 : La "reconquête" du littoral de Sfax et ses enjeux. In Mondialisation et changement urbain, sous la direction de Dlala H., Tunis, Centre de Publication Universitaire, 139-160.

MILLS G., 2007 : Cities as agents of global change. Int. J. Climatol., 27, 1849-1857.

MINISTERE DE L'ENVIRONNEMENT ET DE L'AMENAGEMENT DU TERRITOIRE (MEAT), 1998 : Schéma Directeur d'Aménagement du Grand Sfax. 223 pages.

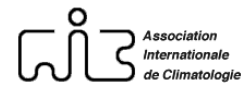


MINISTERE DE L'ENVIRONNEMENT ET DU DEVELOPPEMENT DURABLE (MEDD), 2009a : Rapport National sur l'état de l'environnement. Tunisie, 210 pages.

MINISTERE DE L'ENVIRONNEMENT ET DU DEVELOPPEMENT DURABLE (MEDD), 2009b : Etude d'élaboration de la seconde communication nationale de la Tunisie au titre de la convention cadre des Nations Unies sur les changements climatiques. Phase III, Vulnérabilité de la Tunisie face aux changements climatiques. Tunisie, 85 pages.

MUNICIPALITE DU GRAND SFAX, 2004 : Stratégie de Développement du Grand Sfax, Rapport de synthèse - Phase de diagnostic. 163 pages.

OKE T. R., 1987 : The surface energy budgets of urban areas. In Modelling the urban boundary layer, AMS, 1-52.

TORETI A. et DESIATO F., 2008 : Change in temperature extremes over Italy in the last 44 years. Int. J. Climatol., 28, 733-745. 